\title{
An Analytical Path-Loss Model for On-Body Radio Propagation
}

\author{
G. A. Conway ${ }^{*}$, W. G. Scanlon ${ }^{\# 1}$, S. L. Cotton ${ }^{* 2}$, M. J. Bentum ${ }^{\# 3}$

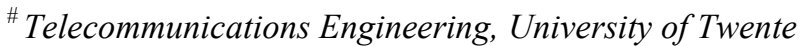 \\ Enschede, The Netherlands \\ w.g.scanlon@utwente.nl \\ ${ }^{3}$ m.j.bentum@ewi.utwente.nl \\ *Institute of Electronics, Communications and Information Technology, \\ Queen's University of Belfast, UK \\ 2simon.cotton@qub.ac.uk
}

\begin{abstract}
An analytical model for across the body surface communication systems is presented. The paper is focused on the calculation of the approximate path gain, both along and around planar and cylindrical geometries, representative of the human body for antennas polarized normal to the body surface. The model is validated at $2.45 \mathrm{GHz}$ using finite-difference timedomain numerical analysis and in-situ measurements on an adult-male test subject.
\end{abstract}

\section{INTRODUCTION}

Understanding electromagnetic interactions with the human body is essential for the development and optimization of body-centric radio communication systems. At UHF frequencies, the human body is an irregularly shaped heterogeneous lossy dielectric medium with frequency dependent permittivity and conductivity. Nonetheless, the complexity of this electromagnetic problem is often simplified by representing the biological body by geometries such as cylinders and spheres of lossy dielectric material, where the body is assumed to be stationary, isotropic and homogeneous. For on-body systems, where both the transmitting and receiving antennas are positioned close to the body surface, there are a number of known wave propagation mechanisms: the direct (space) wave, reflected waves, surface waves and diffracted creeping waves. Analytical models, such as the one presented in this paper, can help aid the understanding of these mechanisms and therefore may lead to improvements in antenna design for on-body systems.

In this paper we consider an electric current source polarized normal to a dielectric medium of finite conductivity, a configuration representative of recent developments in onbody antennas [1]. The approach taken was based on the analytical solutions developed by Norton [2] and Wait [3] for propagation of electromagnetic waves over planar and spherical Earth, respectively, as presented in [4]. However, it is for the first time that these solutions have been directly applied to body-centric communications and developed for both the planar and cylindrical propagation paths found in onbody applications. The analytical model is presented in Section II and is initially validated for planar and cylindrical structures using finite-difference time-domain (FDTD) numerical simulation. Section III presents experimental validation results at $2.45 \mathrm{GHz}$ for an adult-male test subject, which are also compared with FDTD analysis of a whole body numerical phantom. Finally, the measured path gain results were used to derive generic on-body propagation trends from Frii's transmission equation for wearable antennas polarized normal to the surface of the body at $2.45 \mathrm{GHz}$.

\section{ANALYTICAL MODEL}

\section{A. Homogeneous Planar Dielectric Medium}

Propagation along the length of the body, where the curvature of the body is small, may be approximated by considering a planar lossy dielectric half-space (Fig. 1).

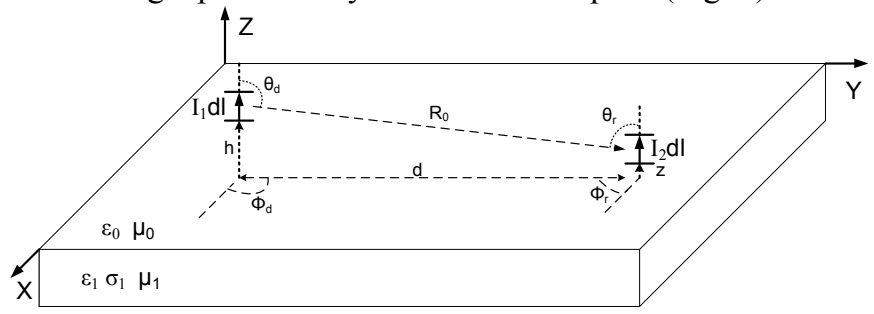

Fig. 1. Geometry for a vertical current element above planar media

The homogeneous medium represents biological tissue and has a relative permittivity $\varepsilon_{1}$, relative permeability $\mu_{1}$ and finite conductivity $\sigma_{1}$. A vertical electric current element $I d l$ excites a transverse magnetic (TM) wave in cylindrical coordinates for which the z-directed electric farfield component, $E_{z}$, at a distance $R_{0}$ obtained from [4] is:

$E_{z}=\frac{-j k_{0} I d l Z_{0}}{4 \pi}\left[\begin{array}{l}\sin ^{2} \theta_{d} \frac{\exp \left(-j k_{0} R_{0}\right)}{R_{0}}+R_{v} \sin ^{2} \theta_{r} \frac{\exp \left(-j k_{0} R_{1}\right)}{R_{1}} \\ +\left(1-R_{v}\right) F(w) \sin ^{2} \theta_{r} \frac{\exp \left(-j k_{0} R_{1}\right)}{R_{1}}\end{array}\right]$

Where the free space wave number $k_{0}=2 \pi / \lambda$, the wave number of the dielectric medium $k_{1}=k_{0}\left(\varepsilon_{r 1}-j 60 \sigma_{1} \lambda_{0}\right)^{1 / 2}$, the reflection coefficient $R_{v}=\cos \theta_{r}-\Delta_{0} / \cos \theta_{r}+\Delta_{0}$, the attenuation function $F(w)=1-j(\pi \omega)^{1 / 2} e^{-w} \operatorname{erfc}\left(j \omega^{1 / 2}\right)$, $w=-j k_{0} R_{1} / \sin ^{2} \theta_{r}\left(\cos \theta_{r}+\Delta_{0}\right)^{2}$, the normalized surface 
impedance $\Delta_{0}=k_{0} / k_{1}\left[1-\left(k_{0} / k_{1}\right)^{2} \sin ^{2} \theta\right]^{1 / 2}$ where $\theta=\pi / 2$ and the complex complementary error function erfc defined as:

$$
\operatorname{erfc}\left(j \omega^{1 / 2}\right)=-j \frac{2}{\pi^{1 / 2}} \int_{-j \infty}^{w^{1 / 2}} e^{u^{2}} d u
$$

Eqn. (1) explicitly contains a direct wave, reflected wave and a surface wave with an associated attenuation function $F(w)$, which is based on the approximation that the wave number in the lossy media is much greater than that of the wave number in free-space $\left|k_{1}\right| \gg\left|k_{0}\right|$. The analytical solution is valid for every far-field point, for any range of height of transmitter or receiver. However, in the context of on-body wearable systems, only source and field points close to the surface of the media are considered.

Using (1), the electric field magnitude from a vertically polarized current source at observation distances $d=0.02$ to $0.45 \mathrm{~m}$ from the source were calculated. Both the source and field points were positioned at a height $h=1 \mathrm{~mm}$ above the biological media with $\varepsilon_{1}=53$ and conductivity $\sigma_{1}=1.8 \mathrm{Sm}^{-1}$, equivalent to muscle tissue at $2.45 \mathrm{GHz}$ [1]. FDTD numerical analysis was used to compute the field values for the same problem geometry, with the results compared in Fig. 2. In the simulation a monopole antenna tuned to be resonant at centre frequency of $2.45 \mathrm{GHz}$ was used to produce an electromagnetic wave polarized normal to planar numerical phantom of finite dimensions $800 \times 600 \times 100 \mathrm{~mm}$. Extending the phantom dimensions did not produce significant changes in results. From the FDTD analysis, vector field components of the E-field were extracted at intervals along the media. The total fields with respect to the unit vector were calculated for comparison with the analytical analysis. At low heights the direct and reflected waves are reduced and the surface wave is the dominant propagation mode. There was good agreement between the analytical and numerical results.

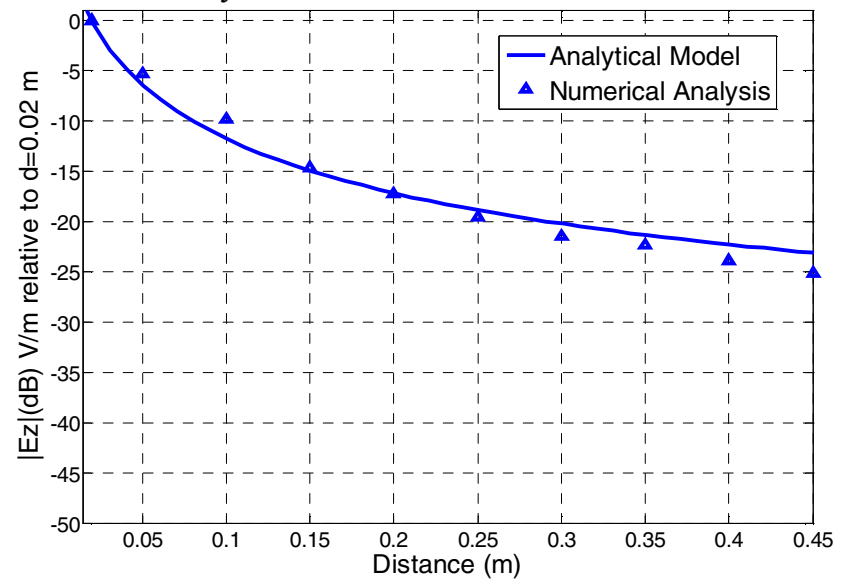

Fig. 2. Comparison of analytical model and FDTD simulation of E-field magnitude at $2.45 \mathrm{GHz}$ due to a vertically polarized current source positioned above planar lossy dielectric media (values relative to a distance of $0.02 \mathrm{~m}$ )

\section{B. Propagation Over Curved Homogeneous Dielectric Media}

To represent propagation around the curvature of the human body we consider the electric field expression for a vertically polarized current source with an attenuation function due to the presence of cylindrical media. In [4], a field expression satisfying the boundary conditions for a source above dielectric media is derived in terms of residue series. Assuming no variation in the E-field component along the length of the cylinder, the E-field in the direction of the unit vector $\boldsymbol{n}$ normal to any point on or off the cylinder surface may be calculated from the radial electric field component $\left|E_{r}\right|$. On a closed curved surface such as a cylinder, there will be an additional field contribution due to a creeping wave which propagates around the cylinder in the reverse direction. The resulting expression for the total electric field due to a current element $I d l$ vertically polarized in the z-direction at a height $h$ for field points on or near the surface of the cylinder with radius $a$ (Fig. 3) is:

$$
\begin{aligned}
& E_{r}=-\frac{j k_{0} a I d l Z_{0}}{4 \pi b} \frac{\exp \left(-j k_{0} a \theta\right)}{d} e^{j\left(\omega_{0}-\pi / 4\right)} 2(\pi x)^{1 / 2} \times \\
& \sum_{s=1}^{\infty} \frac{e^{-j x t_{s}}}{t_{s}-q^{2}} \frac{w_{1}\left(t_{s}-y_{1}\right)}{w_{1}\left(t_{s}\right)} \frac{w_{1}\left(t_{s}-y_{2}\right)}{w_{1}\left(t_{s}\right)} \\
& -\frac{j k_{0} a I d l Z_{0}}{4 \pi b} \frac{\exp \left(-j k_{0} a \theta_{c}\right)}{d} e^{j\left(\omega_{0}-\pi / 4\right)} 2\left(\pi x_{c}\right)^{1 / 2} \times \\
& \sum_{s=1}^{\infty} \frac{e^{-j x t_{s}}}{t_{s}-q^{2}} \frac{w_{1}\left(t_{s}-y_{1}\right)}{w_{1}\left(t_{s}\right)} \frac{w_{1}\left(t_{s}-y_{2}\right)}{w_{1}\left(t_{s}\right)}
\end{aligned}
$$

Where $w_{1}(t)$ denotes the Fock notation of the Airy function. The roots $t_{s}$ satisfy the boundary condition at the lossy tissue surface $w_{1}^{\prime}-q w_{1}(t)=0$, with $C=\left(k_{0} a / 2\right)^{1 / 3}$ and $q=-j C \Delta_{0}$. The roots $t_{s}(q)$ can be found by a Taylor Series expansion at $q=0$, where for small values $|q|<\left|t_{s}^{0}\right|$ :

$$
t_{s}(q)=t_{s}^{0}-\frac{q}{t_{s}^{0}}-\frac{q^{2}}{2\left(t_{s}^{0}\right)^{3}}+\frac{q^{3}}{1 / 3\left(t_{s}^{0}\right)^{2}+1 / 2\left(t_{s}^{0}\right)^{5}} \ldots
$$

Where the roots denoted by $t_{s}{ }^{0}$ for increasing integer values of $s$ can be found from:

$$
t_{s}^{0}=\left|[1.5(s-3 / 4) \pi]^{2 / 3}\right| e^{-j \pi / 3}
$$

Where $s$ is an arbitrarily high value (i.e., $s>1000$ ), $x=C \theta$, $y_{1}=k_{0} h_{1} / C, y_{2}=k_{0} h_{2} / C, x_{c}=C \theta_{c}$ and $\theta_{c}=2 \pi-\theta$. $R_{0}=\left[d^{2}+(h-z)^{2}\right]^{1 / 2}$ is the distance between source at a height $h$ and observation point at a height $z$. The arc distance from the source to the observation point is $d=\theta / a$.

Using Equation (3) the radial electric field component for each point around the surface of a cylinder of radius $a=150 \mathrm{~mm}$ was calculated for $1>\theta \leq \pi$. Both the source and observation points were positioned at height $h=1 \mathrm{~mm}$ above the cylinder which had a relative permittivity $\varepsilon_{1}=53$ and conductivity $\sigma_{1}=1.8 \mathrm{Sm}^{-1}$. FDTD numerical analysis was then used to solve for the magnitude of the electric field component relative to the unit vector $\boldsymbol{n}$ (normal to the surface) for the same problem geometry, assuming a finite cylinder phantom with a length of $0.8 \mathrm{~m}$. The analytical and numerical analysis results are compared in Fig. 4. The analytical results give a very good approximation of the field as a function of distance around cylindrical biological media. 


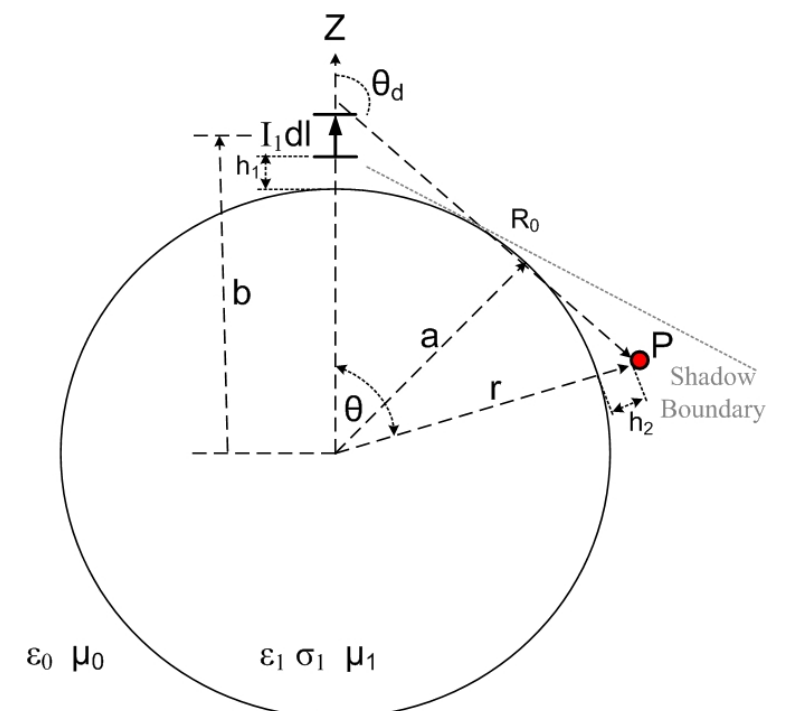

Fig. 3. Geometry for a vertical current element above cylindrical media

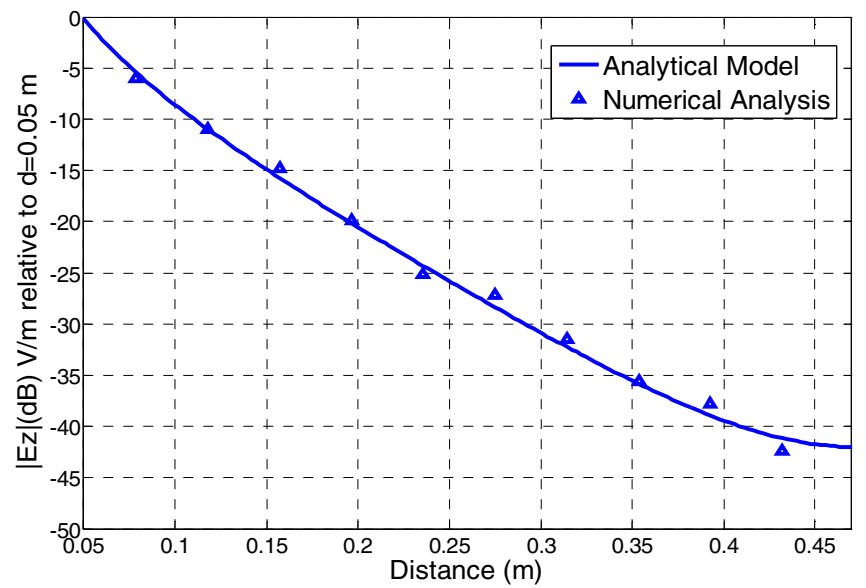

Fig. 4. Comparison of analytical model and FDTD simulation of E-field magnitude at $2.45 \mathrm{GHz}$ due to a normally polarized current source positioned above curved lossy media with a radius of $150 \mathrm{~mm}$ (values relative to a distance of $0.05 \mathrm{~m}$ )

\section{EXPERIMENTAL VALIDATION}

\section{A. Simulation and Measurement Setup}

Whole body FDTD simulations of electromagnetic wave propagation mechanisms along and around the torso of the SEMCAD-X human phantom [5] at $2.45 \mathrm{GHz}$ are shown in Fig. 5. The E-field magnitude cross-sections through the phantom show that a source or antenna radiating close to the surface of the human body produces an electromagnetic wave that travels along and around the surface of the body. The creeping wave propagation mechanism is shown by the electric field component of the wave which propagates around the surface of the media to a receive location on the back of the phantom body. The propagating wave continually diffracts electromagnetic waves at tangents to the surface. The creeping wave attenuation is largely due to this wave diffraction, therefore, only a small percentage of the power is conveyed to the receiver in the shadow region at the back of the torso. From the FDTD analysis, vector field components of the E- field were extracted at point locations at $100 \mathrm{~mm}$ intervals along the front and around the radius of the body (Fig. 5). The total field at these points with respect to the unit vector was calculated for comparison to analytical analysis.

Experimental measurements of $\left|S_{21}\right|$ path gain were taken at corresponding $100 \mathrm{~mm}$ intervals along and around the perimeter of the human body torso using monopole antennas resonant at $2.45 \mathrm{GHz}$. Monopole antennas are equivalent to the electric current source in the analytical model. The antennas were positioned at height $h=3 \mathrm{~mm}$ (the thickness of velcro attachments) from the body surface. The human body subject was a 31 year old male, $1.80 \mathrm{~m}$ tall with a mass of $89.5 \mathrm{~kg}$. The arms were placed alongside the torso as in the numerical phantom model (Fig. 5). The tests were conducted in an anechoic chamber using a Rohde \& Schwarz ZVB8 vector network analyzer for a frequency range of $2-3 \mathrm{GHz}$.

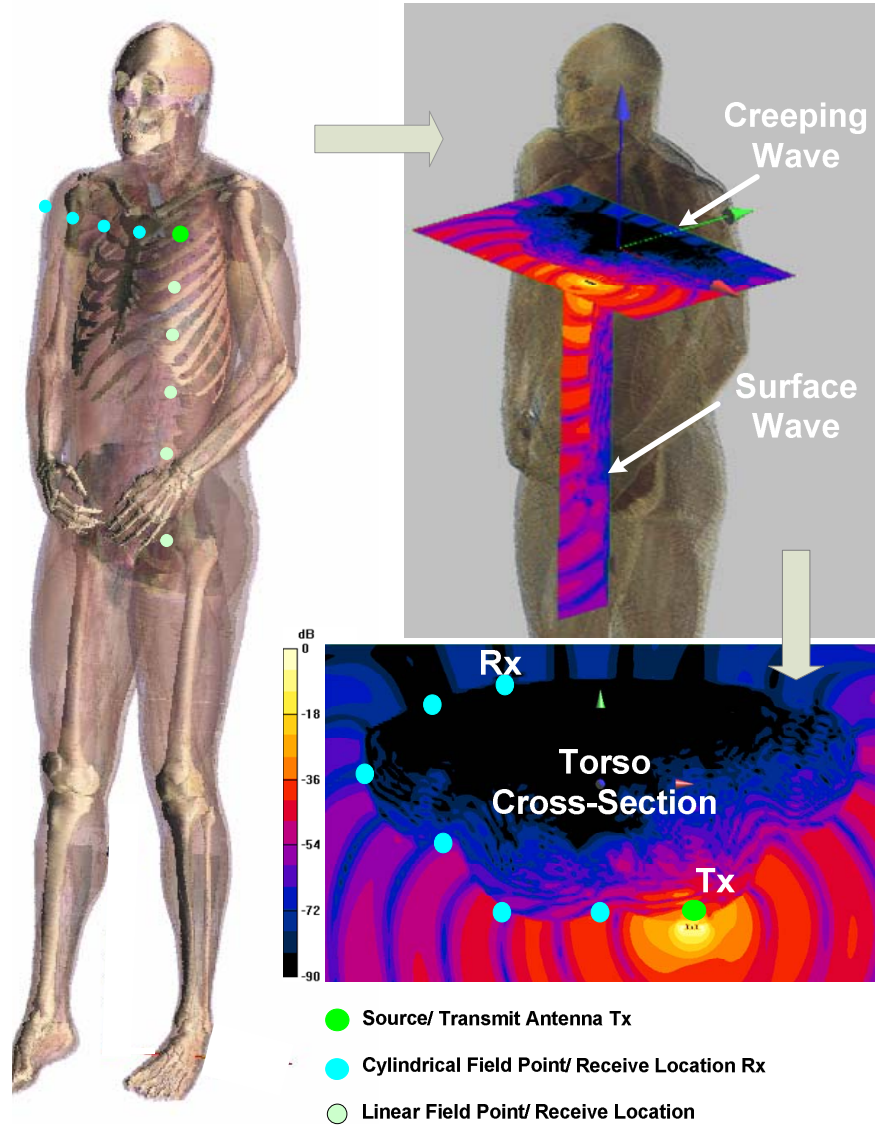

Fig. 5. FDTD simulation of whole body adult male numerical phantom illustrating on-body propagation with E-field magnitude slice data along and around the body with field point measurement locations highlighted

\section{B. Results}

Measured results along the torso are compared with the corresponding FDTD numerical simulation on the whole body phantom and the planar analytical model (Eqn. (1)) in Fig. 6. There was very good agreement between the numerical simulated and measurement results. However, the planar analytical showed lower attenuation than the measurement results for distances greater than $0.25 \mathrm{~m}$. The analytical model assumes that the body is smooth and flat, therefore it does not 
account for the small curvature and irregular geometries along actual human bodies. The curved and irregular geometry of the body increases the scattering and diffraction of the propagating wave in comparison to a smooth flat surface; hence the path gain is reduced. Nonetheless, the analytical model would prove useful in initial communication system design and engineering.

Measurements of the human torso perimeter both on the live subject and the whole body numerical phantom showed that a cylinder of radius $a=200 \mathrm{~mm}$ gave an equivalent torso perimeter path length with the analytical model. Measured results and the corresponding FDTD numerical simulation on the whole body phantom are compared with the cylindrical analytical model (Eqn. (3)) in Fig. 7. There was excellent agreement between the numerical phantom simulated results and measurement. The analytical model provided a good approximation of the path gain around the curvature of the body surface, especially considering the simplified geometry of the model. Some of the measured and numerical E-field values up to a distance $d=0.4 \mathrm{~m}$ are slightly higher than those predicted by the analytical model. These anomalies may be explained by considering that the cross section of the human body is more elliptical than circular. Since the curvature along the front of the body is less than on a cylindrical geometry, the diffraction losses were lower in this region.

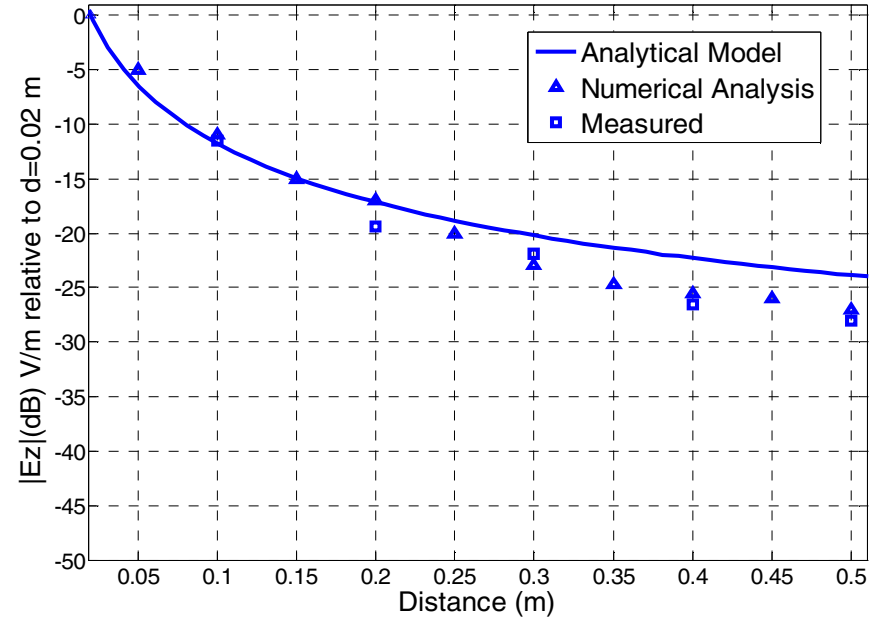

Fig. 6. Comparison of measurements and whole body FDTD numerical analysis with analytical model of E-field magnitude for a vertically polarized current source positioned $3 \mathrm{~mm}$ above planar lossy media (values relative to a distance of $0.02 \mathrm{~m}$ )

\section{IV.PATH LOSS EXPONENT}

The results in Section III can be used to identify general propagation trends using the concept of path loss exponent where the decay index, $n$ is estimated from the results such that $|E|^{2} \propto d^{-n}$. While free space propagation would have a decay index of $n=2$, the results obtained from the wholebody numerical simulations and validation measurements show that along the body surface (planar) transmission has a decay index of $n \approx 2.4-2.5$. However, the increased diffraction losses and non-line of sight conditions for around the body links gives a much higher decay index of $n \approx 4.0-4.5$.

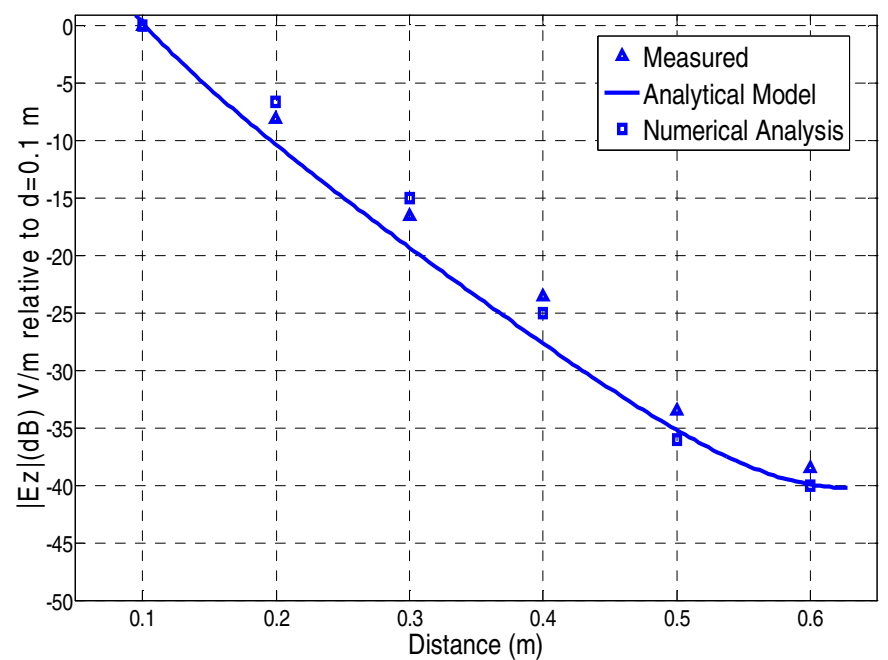

Fig. 7. Comparison of measurements and whole body FDTD numerical analysis with analytical model of E-field magnitude for a vertically polarized source positioned $3 \mathrm{~mm}$ above curved lossy media with radius of $200 \mathrm{~mm}$ (values relative to a distance of $0.1 \mathrm{~m}$ )

It is evident from this analysis that path gain trends vary considerably depending on the propagation mechanism. The location of the observation point relative to the source determines which propagation mechanism is dominant. The on-body propagation scenarios presented in this paper show quite low path gains for what are relatively short distances around the human body. However, recent channel characterisation studies in non-anechoic environments [6] have shown that on-body links can benefit from multipath scattering effects, particularly for around the body (non-line of sight) communication.

\section{CONCLUSION}

Analytical models, valuable for estimating the path gain around the human body were presented and validated using measurement and FDTD numerical analysis. The models are considerably faster and less computationally expensive than FDTD. General propagation trends were identified which may be useful to system designers.

\section{REFERENCES}

[1] G.A. Conway \& W. G. Scanlon, "Antennas for over-body-surface communication at $2.45 \mathrm{GHz}$," IEEE Trans. Antennas \& Propagation, Vol. 57, 4, pp. 844-855, 2009.

[2] K. A. Norton, "Propagation of radio waves over a plane earth," Nature, 135, pp. 954-955, 1935

[3] J. R. Wait, Electromagnetic Waves in Stratified Media. New York: IEEE Press, 1996.

[4] T. S. M. Maclean and Z. Wu, Radiowave Propagation Over Ground, UK: Chapman \& Hall, 1993.

[5] Reference manual for the SEMCAD Simulation Platform for Electromagnetic Compatibility, Antenna Design and Dosimetry, Schmid \& Partner Engineering AG.

[6] S. L. Cotton and W. G. Scanlon, "An experimental investigation into the influence of user state and environment on fading characteristics in wireless body area networks at $2.45 \mathrm{GHz}$," IEEE Trans. Wireless Communications, Vol. 8, 1, pp. 6-12, 2009. 